\title{
Normal mesenteric lymph ameliorates lipopolysaccharide challenge-induced spleen injury ${ }^{1}$
}

\author{
Li-min Zhang, Wen Song ${ }^{\mathrm{I}}$, Hao Cui ${ }^{\mathrm{II}}$, Li-qiang Xing ${ }^{\mathrm{III}}$, Hui-bo Du${ }^{\mathrm{IV}}$, Ying Cui ${ }^{\mathrm{II}}$, Wei-hong Chen ${ }^{\mathrm{II}}, \mathrm{Zi}^{\mathrm{I}}$-gang Zhao ${ }^{\mathrm{IV}}$, Chun-yu Niu ${ }^{\mathrm{V}}$ \\ DOI: http://dx.doi.org/10.1590/S0102-865020150090000004 \\ IMaster, Institute of Microcirculation, Hebei North University, Zhangjiakou, China. Acquisition of data. \\ "Undergraduate, Hebei North University, Zhangjiakou, China. Acquisition of data. \\ III Master, Full Professor, Institute of Microcirculation, Hebei North University, Zhangiiakou, China. Acquisition of data. \\ ${ }^{\mathrm{IV}}$ Bachelor, Experimentalist, Institute of Microcirculation, Hebei North University, Zhangjiakou, China. Acquisition of data, manuscript writing, \\ critical revision. \\ ${ }^{\mathrm{v}} \mathrm{PhD}$, Full Professor, Institute of Microcirculation, Hebei North University, Zhangjiakou, China. Design of the study, manuscript writing, critical \\ revision.
}

\begin{abstract}
PURPOSE: This study was conducted to investigate the effect of normal mesenteric lymph (NML) from mice on the spleen injury induced by lipopolysaccharide (LPS) challenge.

METHODS: Mice in the LPS and LPS+NML groups received an intraperitoneal injection of LPS $(35 \mathrm{mg} / \mathrm{kg})$ and $\mathrm{kept}$ for $6 \mathrm{~h} .$. The mice in the LPS+NML group received NML treatment at $1 \mathrm{~h}$ after LPS injection. Afterward, the splenic morphology, the levels of lipopolysaccharide-binding protein (LBP), cluster of differentiation 14 (CD14), phosphorylation mitogen-activated protein kinases (MAPKs), and inflammatory mediators in splenic tissue were investigated.

RESULTS: LPS injection induced spleen injury, increased the levels of LBP, CD14, tumor necrosis factor- $\alpha$ (TNF- $\alpha$ ), interleukin 6 (IL-6), and interferon $\gamma$ (IFN- $\gamma$ ), and decreased the IL-4 content in the spleen. By contrast, NML treatment reversed these changes. Meanwhile, the LPS challenge decreased the phosphorylation levels of p38 MAPK, extracellular regulated protein kinases $1 / 2$, and c-Jun N-terminal kinase (JNK). Moreover, the phosphorylation levels of p38 MAPK and JNK were further decreased by the NML administration.
\end{abstract}

CONCLUSION: rRdThe normal mesenteric lymph treatment alleviated lipopolysaccharide induced spleen injury by attenuating LPS sensitization and production of TNF- $\alpha$, IL- 6 , and IFN- $\gamma$.

Key words: Lymph. Lipopolysaccharides. Spleen. Inflammation Mediators. Mitogen-Activated Protein Kinases. Histology. Mice. 


\section{Introduction}

Previous studies have shown that the immune system dysfunction plays an important role in inflammation response imbalance and organ injury following shock, trauma, and sepsis $^{1-3}$. The spleen is a major immune organ and is the center of cellular and humoral immunities. Previous studies have reported that lipopolysaccharide (LPS) challenge could cause spleen injury ${ }^{4,5}$. Meanwhile, pre-treatment with interleukin-10 (IL-10) followed by LPS leads to a reduction in splenic TNF- $\alpha$ and LPS-induced spleen injury ${ }^{5}$. In previous studies, the exogenous normal mesenteric lymph (NML) from healthy dog has alleviating effects on LPS-induced microcirculation disturbance and acute lung and kidney injury in rats $^{6-8}$. In addition, NML obtained from mice could ameliorate acute kidney injury induced by the LPS challenge, and such effect is related to decreasing inflammation and LPS sensitization ${ }^{9}$. However, whether or not NML could eliminate LPS-induced spleen injury remains unclear. Therefore, we sought to determine the effectiveness of NML on LPS-induced spleen injury and reveal its mechanism.

\section{Methods}

A total of 36 healthy and specific pathogen-free (SPF) BALC/c mice (20-25g, purchased from the National Institutes for Food and Drug Control, Beijing, China) were used in this study. A total of 18 mice were used for NML drainage for the treatment, whereas the rest was randomly divided into the following: sham, LPS, and LPS+NML groups (six mice in each group). Before the experiment, the mice were subjected to fasting for $12 \mathrm{~h}$, but they were allowed free access to water. All animal procedures were reviewed and approved by the Institutional Animal Care and Use Committee of Hebei North University and conformed to the National Institutes of Health guidelines. All efforts were made to minimize the suffering of the animals during the experiment.

\section{NML harvest}

After anesthetization with intraperitoneal injection of pentobarbital sodium $(1 \%, 50 \mathrm{mg} / \mathrm{kg})$ under the operating microscope (SSJ, Shanghai optical instrument factory, Shanghai, China), the mice received a laparotomy with $2 \mathrm{~cm}$ longitudinal incision on deviation to the right of the midline. Their mesenteric lymph was drained with a drainage needle continuously for $60 \mathrm{~min}$ into a sterile test tube. Lymph sample was centrifuged for $5 \mathrm{~min}$ at $315 \mathrm{~g}$ to remove all cellular components and was stored at $-75^{\circ} \mathrm{C}$ to $-80^{\circ} \mathrm{C}$ for the treatment.

\section{LPS challenge and NML administration}

After anesthetization, the mice were placed under the operating microscope and received a femoral operation for the anticoagulation with heparin sodium $(1 \%, 1 \mathrm{ml} / \mathrm{kg})$ injection via the right femoral artery. The animals' mean artery pressure (MAP) levels were continuously monitored using biological signal collecting and processing system (RM-6240B, Chengdu Inc., Chengdu, China) via the right femoral artery. After a $30 \mathrm{~min}$ stabilization period, the intraperitoneal injection of LPS $(0.5 \%$, $35 \mathrm{mg} / \mathrm{kg}$ ) (Escherichia coli O111:B4) (Sigma, Milwaukee, WI) was performed in the LPS and LPS+NML groups. After $1 \mathrm{~h}$ of LPS injection, NML was injected through the femoral artery in the LPS+NML group. The same amount of saline (instead of NML) was given to a separate LPS group as control. The mice in the sham group received the same operation as described above, but without administering LPS and NML. The same amount of saline instead of LPS or NML was given to the mice in the sham group.

\section{Splenic tissue collection}

At $6 \mathrm{~h}$ after LPS injection, the spleens were immediately obtained from the mice under deep anesthesia. Subsequently, the splenic tissue was divided into two parts. Part one of splenic tissue was fixed in neutral buffered paraformaldehyde of $4 \%$ for morphological observation. Part two of splenic tissue was mixed with nine times the amount of normal saline and was subsequently homogenized for $30 \mathrm{~s}$ and centrifuged at $850 \mathrm{~g}$ at $0{ }^{\circ} \mathrm{C}-4^{\circ} \mathrm{C}$ for $10 \mathrm{~min}$ using Labofuge $400 \mathrm{R}$ supercentrifuge (Thermo Fisher Scientific, San Jose, California). The supernatant fluid was frozen at $-75^{\circ} \mathrm{C}$ to $-80{ }^{\circ} \mathrm{C}$ for the measurement of lipopolysaccharide-binding protein (LBP), cluster of differentiation 14 (CD14), phosphorylation mitogen-activated protein kinases (MAPKs), tumor necrosis factor- $\alpha$ (TNF- $\alpha$ ), and IL-6.

\section{Splenic morphology observation}

The fixed splenic tissue was dehydrated with alcohol gradient, embedded in paraffin, and sectioned at $4 \mu \mathrm{m}$ using a microtome (Shandon Finesse 325, Thermo, Waltham, MA). After hematoxylin and eosin (HE) staining was conducted, morphological changes were observed under light microscope (90-i; Nikon, Tokyo, 
Japan), and then images were taken using an image collection and analysis system (Motic Med 6.0; Beijing, China).

\section{Enzyme-linked immunoadsorbent assay (ELISA) analysis}

The levels of LBP, CD14, TNF- $\alpha$, IL- 6 , interferon $\gamma($ IFN- $\gamma$ ), and IL-4 and the phosphorylation levels of p38 MAPK, extracellular regulated protein kinases (ERK) 1/2, and c-Jun NH2-terminalprotein kinase (JNK) in splenic homogenates were detected by according to the manufacturer's instructions. The mouse-ELISA kit was purchased from Jiangsu Hope Biotechnology Ltd., Zhenjiang, China. Antibodies of LBP, CD14, TNF- $\alpha$, IL- 6 , IFN- $\gamma$, and IL-4 were obtained from R\&D Systems, Minneapolis, MN and antibodies of p-p38 MAPK, p-ERK1/2, and p-JNK were obtained from MolecularTag (USA) using the SpectraMax ${ }^{\circledR}$ M3 Microplate Reader (Molecular Devices, Sunnyvale, CA). Protein concentration in the homogenate was quantified by the Coomassie brilliant blue colorimetric method ${ }^{10}$ according to the instructions provided by the manufacturer (Jiancheng Biotechnology Research Institute, Nanjing, China). Results were normalized by the protein contents in the homogenates.
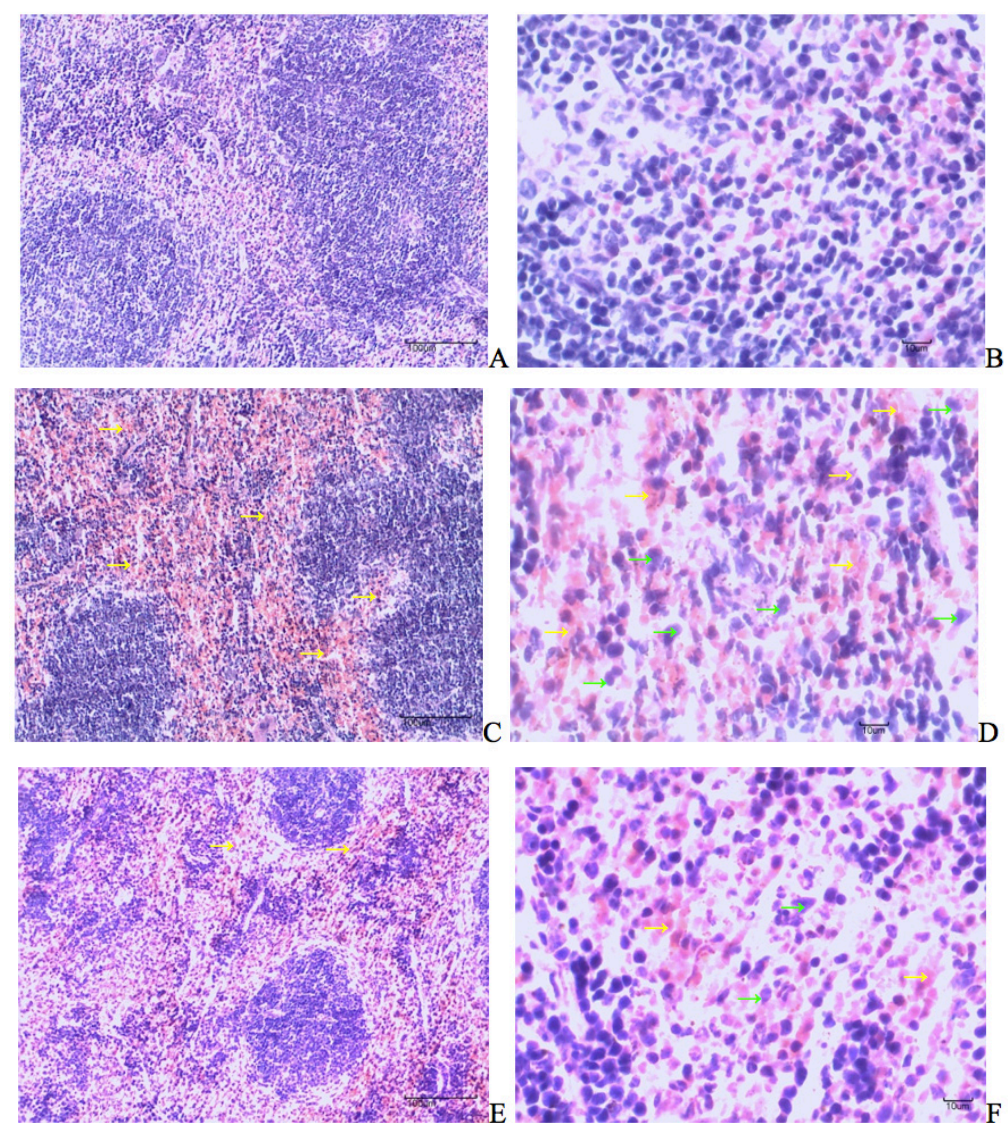

FIGURE 1 - Effect of normal mesenteric lymph (NML) on splenic morphology change in mice (HE staining, A, C, and E: x100; B, D and F: x400). A-B: Sham group; C-D: lipopolysaccharide (LPS) group; E-F: LPS+NML group. The normal structure in splenic tissues of the sham group were observed, as shown on A and B; the congestion in the splenic sinuses (yellow arrows) and swollen splenocytes (green arrows) were found in the LPS group, as shown in $\mathrm{C}$ and $\mathrm{D}$; the lesser congestion (yellow arrows) and swollen splenocytes (green arrows) were appeared in the LPS+NML group, as shown on E and F. 


\section{LBP and CD14 in spleen}

In Figure 2, at $6 \mathrm{~h}$ after LPS intraperitoneal injection, the levels of LBP and CD14 in the spleen of the LPS group significantly increased compared with that of the sham group $(\mathrm{p}<$ 0.05). By contrast, the levels of LBP and CD14 in the spleen of the LPS+NML group significantly decreased compared with that of the LPS group ( $<<0.05)$, and no statistical difference was found compared with the sham group $(\mathrm{p}>0.05)$.

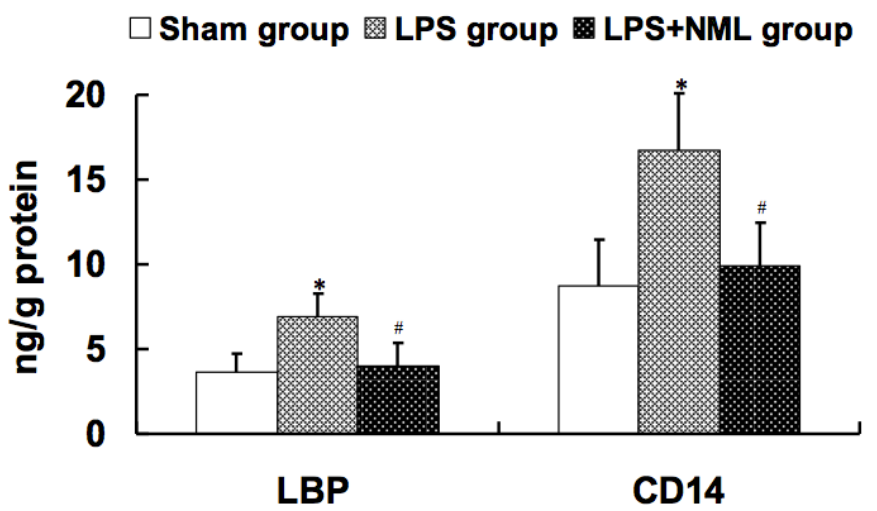

FIGURE 2 - Effects of normal mesenteric lymph (NML) on the levels of lipopolysaccharide-binding protein (LBP) and lipopolysaccharide receptor (CD14) in spleen after lipopolysaccharide (LPS) administration in mice (ng/g protein, mean $\pm \mathrm{SD}, \mathrm{n}=6$ ). ${ }^{*} \mathrm{p}<0.05$ vs. the sham group; \# $p<0.05 v s$. the LPS group.

\section{$T N F-\alpha$ and IL-6 in spleen}

Figure 3 indicated that the LPS administration significantly increased the TNF- $\alpha$ and IL-6 levels in the spleen $(p<0.05)$, and these levels were abolished by the NML injection $(\mathrm{p}<0.05)$. Meanwhile, no statistical difference was found in the TNF- $\alpha$ and IL-6 levels of the LPS+NML and sham groups ( $p>0.05)$.

\section{$\square$ Sham group 图 LPS group 종 LPS+NML group}



FIGURE 3 - Effects of normal mesenteric lymph (NML) on the levels of tumor necrosis factor- $\alpha$ (TNF- $\alpha$ ) and interleukin-6 (IL-6) in spleen after lipopolysaccharide (LPS) administration in mice (ng/g protein, mean $\pm \mathrm{SD}$, $\mathrm{n}=6) .{ }^{*} p<0.05 v s$. the sham group; ${ }^{*} p<0.05 v s$. the LPS group.

\section{MAPKs in spleen}

At $6 \mathrm{~h}$ after LPS injection, the levels of p-p38 MAPK, p-ERK1/2, and p-JNK significantly decreased compared with those of the sham group $(\mathrm{p}<0.05)$. At the same time, the NML treatment further decreased the levels of p-p38 MAPK and p-JNK $(p<0.05)$, along with decreases in the p-p38 MAPK, p-ERK1/2, and p-JNK levels compared with the sham mice ( $\mathrm{p}<0.05$; ; Figure 4$)$.



FIGURE 4 - Effects of normal mesenteric lymph (NML) on the phosphorylation levels of p38 mitogen-activated protein kinases (MAPK), extracellular regulated protein kinases (ERK) 1/2, and c-Jun NH2-terminalprotein kinase (JNK) in spleen after lipopolysaccharide (LPS) administration in mice (ng/mg protein, mean $\pm \mathrm{SD}, \mathrm{n}=6){ }^{*} p<0.05$ $v s$. the sham group; ${ }^{\#} p<0.05 v s$. the LPS group.

\section{$I N F-\gamma$ and IL-4 in spleen}

LPS administration significantly increased the INF- $\gamma$ content and decreased the IL-4 content, thereby resulting in a higher ratio of INF- $\gamma / \mathrm{IL}-4$ in the spleen than in the sham group $(\mathrm{p}<0.05)$. However, the NML injection significantly reversed the negative effects induced by LPS injection $(\mathrm{p}<0.05)$. Moreover, NML injection increased IL-4 level and decreased in INF- $\gamma / \mathrm{IL}-4$ compared with the levels in the sham group $(\mathrm{p}<0.05)$.

\section{Discussion}

In the present study, firstly, we observed the NML from normal mice on LPS attack-induced spleen injury, and found that spleen tissue presented with lot of congestion in the red pulp and expanded splenic sinus in mice at $6 \mathrm{~h}$ after LPS administration, which was attenuated by NML treatment. This finding suggested that NML treatment played a protective effect on spleen injury induced by LPS intraperitoneal injection. 



FIGURE 5 - Effects of normal mesenteric lymph (NML) on the levels of interferon- $\gamma$ (INF- $\gamma$ ) and interleukin-4 (IL-4) in splenic tissue in mice after lipopolysaccharide (LPS) administration ( $\mathrm{ng} / \mathrm{g}$ protein, mean $\pm \mathrm{SD}, \mathrm{n}=6$ ). ${ }^{*} \mathrm{p}<0.05 v s$. the sham group; ${ }^{*} \mathrm{p}<0.05 v s$. the LPS group.

In general, inflammatory response is associated with multiple organ injuries during endotoxemia. Several studies suggested that increased expression of LBP and activation of CD14, a LPS receptor, could enhance the sensitization of inflammatory cells to the endotoxin or LPS and link the inflammatory response to organ injuries ${ }^{11-13}$. Tsai and colleagues found that bile duct ligation upregulates CD14 and TNF- $\alpha$ mRNA expressions in splenic tissue and leads to spleen injury ${ }^{14}$. In the present study, NML treatment decreased the LPS injection-induced increases in LBP and CD14 in splenic tissue, which suggests that treatment with NML lessens LPS sensitization. Subsequently, we observed the roles of LPS challenge and NML treatment on the levels of TNF- $\alpha$ and IL- 6 , the most important pro-inflammatory cytokines ${ }^{15}$. The current results showed that LPS administration increased the TNF- $\alpha$ and IL-6 levels in splenic tissue, which were significantly ameliorated by NML treatment. In conclusion, the alleviation of NML treatment on LPS-induced spleen injury is related to lessening the levels of sensitization to LPS and the mediated release of pre-inflammatory mediators.

During the LPS stimulation leading to inflammatory response through binding to LBP and CD14, several intracellular signaling pathways coordinate the induction of many genes encoding inflammatory mediators, such as MAPK pathway: p38 MAPK, ERK $1 / 2$ and JNK, and IkappaB kinase -nuclear factor kappa B (NF-kB) pathway ${ }^{16}$. Meng et al. ${ }^{17}$ reported that LPS administration significantly increased the activations of p38 
MAPK and TNF- $\alpha$ in spleen. Mormède et al. ${ }^{18}$ also illustrated that intraperitoneal injection of LPS induced an activation of the genes coding for NF- $\mathrm{KB}$ and MAPK signaling pathways in the spleen. The present results have shown that the NML treatment decreased the p-p38 MAPK and p-JNK levels in the spleen, which is beneficial to reduce the inflammation. However, the LPS-induced decreases in the p-p38 MAPK, p-ERK1/2, and p-JNK levels, which is different from the above references. Whether or not the decrease in MAPKs levels was related to LPS-induced immune suppression is not clear and needs observation. In addition, NF- $\mathrm{B}$ is the other main signal pathway, which induces the expression of pro-inflammatory mediators. However, whether or not the decrease of the pro-inflammatory mediators in spleen because of NML is related to NF- $\mathrm{KB}$ signal pathway needs further investigation.

Th1 and Th2 cells are two main forms of helper T (Th) cells. Th1 cells produce IFN- $\gamma$, which mediates cellular immune response. Th2 cells produce IL-4, IL-5, IL-13, and IL-25, which mediate humoral immunity. In the physiological state, Thl and Th2 subsets adjust each other to achieve dynamic equilibrium and thus maintain balance in immune functions ${ }^{19,20}$. Spleen is an important immune organ containing a lot of $\mathrm{T}$ lymphocytes. Therefore, to observe the change in immune function and equilibrium of Th1 and Th2 cells following LPS intraperitoneal injection, we examined the changes in IFN- $\gamma$ and IL-4 levels in the spleen and found that LPS administration increased the IFN- $\gamma$ level and the IFN- $\gamma$ / IL-4 ratio. Moreover, the IL-4 level decreased because of LPS administration. These results indicated that Th1 cellular immune response was activated and Th2-type response was suppressed in the mice exposed to the LPS challenge. Reversely, NML treatment reduced the levels of IFN- $\gamma$ and IFN- $\gamma /$ IL- 4 and increased the IL-4 level, which were very advantageous for reducing and delaying the Th2 to Th1 drift, thereby restoring immune balance.

\section{Conclusion}

Normal mesenteric lymph treatment alleviated lipopolysaccharide induced spleen injury by attenuating of sensitization to LPS and decreasing the production of TNF- $\alpha$, IL6 , and IFN- $\gamma$.

\section{References}

1. Boontham P, Chandran P, Rowlands B, Eremin O. Surgical sepsis: dysregulation of immune function and therapeutic implications. Surgeon. 2003 Aug;1(4):187-206. PMID: 15570763.

2. Jiang LN, Yao YM, Sheng ZY. The role of regulatory T cells in the pathogenesis of sepsis and its clinical implication. J Interferon Cytokine Res. 2012 Aug;32(8):341-9. doi: 10.1089/ jir.2011.0080.

3. Ni Choileain N, Redmond HP. Cell response to surgery. Arch Surg. 2006 Nov;141(11):1132-40. doi: 10.1001/archsurg.141.11.1132.

4. Sabarirajan J, Vijayaraj P, Sarkar M, Nachiappan V. Effect of lipopolysaccharide on alteration of phospholipids and their fatty acid composition in spleen and thymus by in vitro metabolic labeling. J Appl Toxicol. 2013 Jun;33(6):418-25. doi: 10.1002/jat.1752.

5. Nandi D, Mishra MK, Basu A, Bishayi B. Effects of IL-18 and IL10 pre-treatment on the alteration of endogenous cytokines in liver and spleen of mice with experimental endotoxemia. Indian J Exp Biol. 2010 Nov;48(11):1103-10. PMID: 21117450.

6. Zhang LL, Zhao ZG, Niu CY, Zhang J. Interference effects of lymph plasma on endotoxic shock in rats. Zhongguo Ying Yong Sheng Li Xue Za Zhi. 2011;27(1):75-80. PMID: 21560348.

7. Zhao ZG, Niu CY, Zhang LL, Zhang J, Han R, Zhang YP, Hou YL. Exogenous normal lymph alleviates lipopolysaccharide-induced acute kidney injury in rats. Ren Fail. 2013;35(6):806-11. doi: 10.3109/0886022X.2013.794680.

8. Zhang LL, Zhao ZG, Niu CY, Zhang J. Exogenous normal lymph alleviates lipopolysaccharide-induced acute lung injury through lessening the adhesion molecules. Acta Cir Bras. 2014 May;29(5):287-91. doi: http://dx.doi.org/10.1590/S010286502014000500001

9. Zhao ZG, Zhang LM, Song W, Du HB, Cui H, Niu CY. Normal mesenteric lymph ameliorates acute kidney injury following lipopolysaccharide challenge in mice. Ren Fail. 2014 Sep;36(8):1304-9. doi: 10.3109/0886022X.2014.938585.

10. Bautista J, Corpas R, Ramos R, Cremades O, Gutiérrez JF, Alegre $\mathrm{S}$. Brain mitochondrial complex I inactivation by oxidative modification. Biochem Biophys Res Commun. 2000 Sep;275(3):8904. doi: 10.1006/bbrc.2000.3388.

11. Fang WH, Yao YM, Shi ZG, Yu Y, Wu Y, Lu LR, Sheng ZY. Effect of recombinant bactericidal/permeability-increasing protein on endotoxin translocation and lipopolysaccharide-binding protein/ CD14 expression in rats after thermal injury. Crit Care Med. 2001 Jul;29(7):1452-9. PMID: 11445707.

12. Fang H, Liu A, Dirsch O, Dahmen U. Liver transplantation and inflammation: is lipopolysaccharide binding protein the link? Cytokine. 2013 Oct;64(1):71-8. doi: 10.1016/j.cyto.2013.07.025.

13. Fang H, Liu A, Sun J, Kitz A, Dirsch O, Dahmen U. Granulocyte colony stimulating factor induces lipopolysaccharide (LPS) sensitization via upregulation of LPS binding protein in rat. PLoS One. 2013 Feb;8(2):e56654. doi: 10.1371/journal.pone.0056654.

14. Tsai $\mathrm{CH}$, Yeh $\mathrm{CH}$, Sheen-Chen SM, Huang CY, Liu YW, Huang CC, Shen SC, Tang RP. The kinetic expression of lipopolysaccharide-binding protein and CD14 gene in obstructive jaundice. J Invest Surg. 2015 Feb;28(1):18-23. doi: 10.3109/08941939.2014.943858

15. Myers MJ, Farrell DE, Palmer DC, Post LO. Inflammatory mediator production in swine following endotoxin challenge with or without co-administration of dexamethasone. Int Immunopharmacol. 2003 Apr;3(4):571-9. doi: 10.1016/S1567-5769(03)00048-1.

16. Guha M, Mackman N. LPS induction of gene expression in human monocytes. Cell Signal. 2001 Feb;13(2):85-94. doi: 10.1016/S08986568(00)00149-2.

17. Meng AH, Ling YL, Zhang XP, Zhao XY, Zhang JL. CCK-8 inhibits expression of TNF-alpha in the spleen of endotoxic shock rats and signal transduction mechanism of p38 MAPK. World J Gastroenterol. 2002 Feb;8(1):139-43. PMID: 11833090.

18. Mormède C, Palin K, Kelley KW, Castanon N, Dantzer R. Conditioned taste aversion with lipopolysaccharide and peptidoglycan does not activate cytokine gene expression in the spleen and hypothalamus of mice. Brain Behav Immun. 2004 Mar;18(2):186-200. doi: 10.1016/ S0889-1591(03)00133-8. 
Zhang L et al.

19. Bobat S, Flores-Langarica A, Hitchcock J, Marshall JL, Kingsley RA, Goodall M, Gil-Cruz C, Serre K, Leyton DL, Letran SE, Gaspal F, Chester R, Chamberlain JL, Dougan G, López-Macías C, Henderson IR, Alexander J, MacLennan IC, Cunningham AF. Soluble flagellin, FliC, induces an Ag-specific Th2 response, yet promotes T-bet-regulated Th1 clearance of Salmonella typhimurium infection. Eur J Immunol. 2011 Jun;41(6):1606-18. doi: 10.1002/ eji.201041089.

20. Qin Q, Liu P, Liu L, Wang R, Yan N, Yang J, Wang X, Pandey M, Zhang JA. The increased but non-predominant expression of Th17and Th1-specific cytokines in Hashimoto's thyroiditis but not in Graves' disease. Braz J Med Biol Res. 2012 Dec;45(12):1202-8. doi: 10.1590/S0100-879X2012007500168.

\section{Correspondence:}

Chun-yu Niu and Zi-gang Zhao

Institute of Microcirculation, Hebei North University

Zhangjiakou Hebei, 075000, China

Phone: (86)0313-4029168 / 313-4029223

ncylxf@126.com

.lymphatics@126.com

zzghyl@126.com

Received: May 5, 2015

Review: July 6, 2015

Accepted: Aug 10, 2015

Conflict of interest: none

Financial source: Foundation of Hundred Innovative Talents in Universities of Hebei Province (BR2-105)

${ }^{1}$ Research performed at Institute of Microcirculation, Hebei North University, Zhangjiakou, China. 